(2) Open Access Full Text Article

\title{
Autism and exergaming: effects on repetitive behaviors and cognition
}

\author{
Cay Anderson-Hanley \\ Kimberly Tureck \\ Robyn L Schneiderman \\ Department of Psychology, Union \\ College, Schenectady, NY, USA
}

This article was published in the following Dove Press journal:

Psychology Research and Behavior Management

15 September 2011

Number of times this article has been viewed

\begin{abstract}
Autism is a neurodevelopmental disorder that leads to impairment in social skills and delay in language development, and results in repetitive behaviors and restricted interests that impede academic and social involvement. Physical exercise has been shown to decrease repetitive behaviors in autistic children and improve cognitive function across the life-span. Exergaming combines physical and mental exercise simultaneously by linking physical activity movements to video game control and may yield better compliance with exercise. In this investigation, two pilot studies explored the potential behavioral and cognitive benefits of exergaming. In Pilot I, twelve children with autism spectrum disorders completed a control task and an acute bout of Dance Dance Revolution (DDR); in Pilot II, ten additional youths completed an acute bout of cyber cycling. Repetitive behaviors and executive function were measured before and after each activity. Repetitive behaviors significantly decreased, while performance on Digits Backwards improved following the exergaming conditions compared with the control condition. Additional research is needed to replicate these findings, and to explore the application of exergaming for the management of behavioral disturbance and to increase cognitive control in children on the autism spectrum.
\end{abstract}

Keywords: autism, repetitive behaviors, exergaming, exercise, executive function

\section{Introduction}

Autistic spectrum disorder (ASD) is a neurodevelopmental disorder that impairs social skills, delays language development, and results in repetitive behaviors and restricted interests which impede academic and social involvement. ${ }^{1}$ ASD now afflicts one out of every 150 children and one in 94 boys in the United States. ${ }^{2}$ Scientific evidence has shown that ASD is based on neurophysiological abnormalities, although it continues to be diagnosed on the basis of observable behaviors. ${ }^{3}$ Central to the diagnosis of the disorder is extreme social isolation and repetitive, even obsessive, attempts to preserve sameness (eg, lining up objects), and to self-stimulate (eg, spinning). ${ }^{1,4}$ Above and beyond the limitations caused by deficits in social communication, repetitive behaviors pose an extra barrier to social interaction and community integration. ${ }^{5}$ Researchers and clinicians have focused on developing effective interventions for decreasing the frequency and severity of problematic stereotypic behaviors, and treatment is often complex, ranging from psychopharmacologic interventions to time-intensive behavior management schedules. ${ }^{6}$

A prominent theory to explain repetitive behaviors in ASD is that they are a symptom of executive dysfunction. ${ }^{4}$ Lopez and colleagues ${ }^{7}$ investigated the relationship between performance on executive function tests and presence of repetitive
Correspondence: Cay Anderson-Hanley Department of Psychology, Union College, Schenectady, NY 12308, USA

$\mathrm{Tel}+\mathrm{I} 5183886355$

Fax +I 5185817260

Email andersoc@union.edu 
behaviors in adults with autism spectrum disorder. They found that the presence of repetitive behaviors is strongly correlated with a tendency to perseverate, which supports the theory that these behaviors are a symptom of executive dysfunction. Individuals with ASD were also found to have significant planning deficits. The theory is that the autistic individual is experiencing executive dysfunction, leaving him or her unable to produce, plan, and manage behaviors in the normal manner. ${ }^{4}$ This inability to control behavior is a possible explanation for the restricted and repetitive nature of the behaviors; the source of executive dysfunction in individuals with ASD may be abnormal frontal lobe development and functioning. ${ }^{?}$

Physical exercise has shown promise for decreasing repetitive behaviors in autistic children, ${ }^{8,9}$ and physical exercise has been shown to improve cognitive function, especially executive function, in normative individuals. ${ }^{10-12}$ The neurophysiological mechanisms by which cognitive functions may be improved by exercise are only partially understood and include immediate and direct improvement in cerebral blood flow, as well as longer term benefits to cerebrovasculature, both of which lead to improved delivery of oxygen and nutrients, as well as removal of byproducts. ${ }^{11}$ In addition, research continues to explore the longer term consequences of exercise for brain health and cognitive function by examining biomarkers indicative of neurotrophic growth, such as brain-derived neurotrophic growth factor, and also via neuroimaging which has suggested neurogenesis, given enlarged structures in the brain after exercise, such as the anterior cingulate cortex, which could impact executive function. ${ }^{13,14}$

It is possible that an improvement in executive control functions in those with ASD could work together with direct benefits of physical exercise and may indicate a mechanism behind increased control of repetitive behaviors. Early observational research demonstrated that five children with autism exhibited a decrease in self-stimulatory behaviors in the classroom, following bouts of exercise during gym and recess, but no decrease was seen after a television-watching control condition..$^{15}$ Kern and colleagues ${ }^{16}$ examined the influence of antecedent jogging, ball-playing, and academic responding on self-stimulation in seven children with autism; only the jogging intervention consistently decreased self-stimulatory behavior in the post-intervention observation period. Powers and colleagues ${ }^{17}$ found a similar effect of just 10 minutes of roller skating as the antecedent condition in a single case observation of an autistic boy. Preliminary evidence was compelling, causing some to call for prescription of exercise as treatment for repetitive behaviors. ${ }^{18}$
Similarly, Rosenthal and Mitchell ${ }^{19}$ found that, among five children with ASD, self-stimulatory behaviors in the classroom decreased after 20 minutes of jogging compared with no precondition. It has been suggested that the decrease in self-stimulatory behaviors might be the result of fatigue; however, this theory has been criticized because studies have shown that physical activity not only decreases repetitive behaviors, but also has positive effects on attention and academic responding of children with autism. ${ }^{17,19}$ These studies suggest that the incorporation of routine breaks for physical exercise throughout the school day for children with ASD could decrease problematic behaviors. However, in each of these studies, the samples were small, even a single case study, and many lacked a control group or comparison condition. Furthermore, effects were only evaluated during the brief period immediately following exercise, thus additional research is needed.

Some studies have also examined the potential impact of longer term exercise for ASD. $\mathrm{Pan}^{20}$ used a within-subjects crossover design for 16 children with ASD and found improved social skills after 10 weeks of group aquatic exercise. However, in another study after 14 weeks of aquatic exercise in seven children with ASD and five controls there were no significant differences found between the two groups, most likely due to limited power, although the pilot study did demonstrate feasibility. ${ }^{21}$ Additionally, in these studies, exercise interventions and repeated socialization opportunities were confounded; future research could refine the design to clarify key factors.

While research on the benefits of physical exercise is encouraging, it may also be possible to derive cognitive benefits from videogame play. Research has demonstrated that under some conditions, videogames serving as computerized mental exercise, can improve cognitive functions in the normative population, but the generalizability of these benefits to everyday functions is debated. ${ }^{22}$ More widely applicable benefits have been noted for such computer training interventions in persons with schizophrenia, a disorder which shares some similar cognitive and behavior challenges with ASD. ${ }^{23,24}$ Videogames and virtual reality exergames (eg, Wii) are widely utilized by youth today and specifically, are often attractive to children with pervasive developmental disorders such as ASD; while there are drawbacks to too much or certain types of videogame play, there are also a number of potential benefits in ASD. ${ }^{25,26}$ For example, there is growing research literature demonstrating the benefits of virtual reality computer programs for improving social skills in ASD. ${ }^{27}$ The focus herein is on the use of virtual realityenhanced exercise for impacting repetitive behaviors and 
cognitive function, specifically executive function, which could add to the control of repetitive behaviors.

Research on virtual reality exergames has been increasing in part given the rise in childhood obesity, and the potential for exergames to increase physical activity since game play is controlled by the physical movements of the player. ${ }^{28}$ The popularity and physical activity potential of exergames such as Wii (eg, bowling and tennis) or Dance Dance Revolution (DDR) has led some school districts to incorporate exergames into their physical education curricula. ${ }^{29}$ The games provide an alternate means of physical activity that may increase exercise participation with the aim of improved fitness. It has been shown that exergames can increase energy expenditure in normative children, ${ }^{30}$ and can also elicit fitness gains in persons with developmental disabilities. ${ }^{31}$ Mhurchu and colleagues ${ }^{32}$ conducted a 12-week study using the EyeToy and found that exergaming led children to play fewer videogames overall and engage in more physical activity per week. Yin and Graham ${ }^{33}$ explored components of DDR, such as music and fun game-like features that may facilitate increased exercise effort through distraction from the discomforts of exercise. Leininger and colleauges $^{34}$ found a higher rating of enjoyment after exergaming using DDR than after traditional treadmill walking. These studies suggest that exergaming can increase exercise behavior, and thus could become a valuable alternative to traditional modes of exercise. Since compliance with exercise can be challenging at any age, but especially among clinical populations such as ASD, ${ }^{18,35}$ exergaming holds particular promise as an exercise intervention given the research noted above on increased motivation, enjoyment, and energy expenditure. However, no prior research has explored the behavioral or cognitive effects of exergaming for persons with autism.

The purpose of the current research is to assess the effects of an acute bout of exergaming on the repetitive behaviors and cognitive performance of children with autism. Previous research suggests that vigorous, aerobic activity decreases repetitive behaviors in the short-term. Literature also suggests that exercise and videogames independently improve performance on tasks assessing executive functioning. Thus, merging exercise and videogaming may have beneficial effects on multiple aspects of dysfunction in children with autism. The current investigation moves away from the case study methodology employed in previous studies, ${ }^{15-17}$ and uses a larger sample across two pilot studies, with some control in a mixed within and between subjects design. We hypothesized that the combination of physical and mental exercise in an exergame would yield behavioral and cognitive benefit. In two pilot studies we explored the potential behavioral and cognitive benefits of a single bout of exergaming. We hypothesized that for youth with ASD, a single bout of exergaming would yield decreased repetitive behaviors and increased executive function, compared with a control condition.

\section{Methods \\ Pilot I: DDR \\ Recruitment}

Participants were students attending a school in the northeastern United States and were recruited through a letter sent to parents by a school administrator. One hundred and eighty students were attending the school's extended school year program during the summer, and letters were sent to the parents of 32 students who met the criteria of being diagnosed with an autism spectrum disorder. Inclusion criteria were that students possess some expressive language, and parents could read and write in English for completion of questionnaires. Fourteen interested participants and their parent or guardian signed an informed consent form. One student elected to drop out of the study before it started and another was unable to attend the 2 sessions required by the study during the 2-week testing period. The Union College Human Subjects Research Committee approved the study.

\section{Measures}

\section{Autism spectrum characterization}

The Gilliam Autism Rating Scale, 2nd edition (GARS-2), ${ }^{36}$ was used to assess the severity of autistic disorder in the participants for research purposes and to provide a standard diagnostic criterion. The GARS-2 was designed to identify and diagnose autism in individuals between the ages of 3 and 22 years. The survey consists of 42 items pertaining to observable and measurable behaviors and associated features of autism. The scale aims to differentiate autism from other conditions with some similarities in presentation, such as Rhett's disorder, Asperger's syndrome, childhood disintegrative disorder, mental retardation, and language disorders. The items are divided into three subscales of 14 items each focusing on stereotyped behaviors, communication, or social interaction. All items are scored on a four-point Likert scale ranging from (0) "never observed" to (3) "frequently observed," meaning the individual behaves in this manner at least 5-6 times per 6-hour period. For the purposes of this study, the survey was completed by the parent or guardian of each participant (via postage paid return envelope). A parent or guardian rated delays in social interactions and language and abnormal functioning in social interaction, 
language, and imaginative play during the first 3 years of the child's life. These "yes" or "no" questions are in accord with the diagnostic criteria for autism from DSM-IV-TR. ${ }^{1}$ The final section of the GARS-2 poses key questions that can be helpful in reaching a diagnostic conclusion and gaining insight on the behaviors that are most characteristic of the individual in question.

\section{Behavioral assessment}

To measure any change in repetitive behaviors, the participants were videotaped interacting while playing (with Legos or Play-Doh) for 5 minutes before and after both the control and exercise tasks. The videotapes were coded according to the repetitive behavior scale of the GARS- $2^{36}$ (for more detailed information regarding the application of the GARS-2 for coding behavioral disturbances, please contact the corresponding author). The videotapes of the sessions were divided into 15-second intervals and a rater noted which, if any, of the target behaviors occurred during that time interval. Higher scores indicate greater prevalence of the behavior.

\section{Executive function}

Digit Span Forward and Backward were both administered in standardized fashion to measure executive function captured in the backward performance. ${ }^{37}$ In the forward task, the participant listened to a string of numbers and repeated them in the same order. In the backward task, the participant listened to a string of numbers and repeated them in reverse order (ie, if the participant is read " $9-4-5$ " he or she would respond "5-4-9"). The string length was increased stepwise and the task discontinued after two failures of the same string length. The number of correct strings repeated is the score, thus higher scores indicate better performance.

The Color Trails Test ${ }^{38}$ measures aspects of executive function, including task-switching. Color Trails 1 requires connecting numbers that are randomly distributed across a page, in order from 1 to 25 . Color Trails 2 consists of two sets of numbers from 1 to 25 . One set is highlighted in pink, while the other is highlighted in yellow. The participant must connect the numbers in order, while alternating colors. Time to complete the task is the outcome, with shorter times indicating better performance.

The Stroop task was administered in a brief 40-item form, ${ }^{39}$ in standardized fashion (A, B, and C trials), to extract from the interference trial (Stroop C) a measure of executive function, including inhibition of response. Stroop A requires the participant to name the color of small blocks printed on a page. Stroop B requires the participant to read typed color names printed in black ink. Stroop C requires the participant to ignore the typed color name and say the color of the ink each word is printed in, which contrasts with the typed color name. Time to complete the task is the outcome, with shorter times indicating better performance.

\section{Procedures}

To test the hypotheses, a within-subjects experimental design was employed, wherein participants were exposed to both the control and experimental condition in an A-B sequential design.

\section{Control session}

During the initial session, participants were exposed to a placebo condition, keeping time and tests consistent with the subsequent exergaming condition, which would allow for some controlled comparison given attention and practice effects known to accompany serial assessments. In the control condition, participants were first videotaped playing for 5 minutes (or later behavioral coding per above). Next, the executive function tests were administered. Participants then watched a 20-minute video of a previously recorded school talent show. After watching the brief show, participants' interactions while playing were again videotaped for 5 minutes. After the play interval, the executive function tasks were re-administered using alternate forms.

\section{Exergaming session}

During the exergaming session which occurred a week later, participants were videotaped while playing for 5 minutes, followed by alternate versions of the executive function tests. Then, participants were introduced to DDR which had a sensor mat interconnected with a gaming system. The mat was positioned in front of a television where dance moves were displayed for the participant to imitate. Instructions were provided in a step-by-step demonstration song included at the beginning of the exergame. All participants exercised for the entire 20-minute period. The score was displayed on the screen and vocal encouragement was given to all participants. After completing the exergame, participants were again videotaped playing for another 5 minutes, and alternate forms of the executive function tests were administered. Upon completion of the final evaluation, participants were given a small toy to thank them for their participation.

\section{Pilot II: Cybercycling}

Data from ten additional youth with ASD were used to examine whether an alternate exergame would produce similar 
behavioral and cognitive effects. These participants were recruited from the community by way of announcements sent to ASD organizations and posted in ASD newsletters. Inclusion criteria were the same as above and the Union College Human Subjects Research Committee approved the study. The measures administered were largely the same as in Pilot I above, including the GARS-2, coded repetitive behaviors, Digit Span Backwards, and Stroop C. Only the Color Trails 2 was not available for comparison. The exergaming intervention was a 20-minute bout using what we refer to as a "cybercycle" (Expresso 3R); which consists of a recumbent stationary bike, interconnected with a videogame (Dragon Chase) displayed on a computer screen. The object of the videogame is to move, by pedaling and steering, around an open landscape chasing floating coins and dragons, and earning points based on speed and color matching. As specified above, behaviors were coded while playing for 5 minutes before and after the exergame, and executive function was also measured pre- and post- with alternate forms.

\section{Results}

\section{Pilot I: DDR}

Data were analyzed from twelve participants (mean age = 14.8 years; standard deviation $[\mathrm{SD}]=2.7$; range: $10-18$ years; eight were male). The average score on the GARS-2 was 70.7 $(\mathrm{SD}=14.4)$. To assess the effect of the DDR exergaming intervention, four repeated measures analyses of variance were performed to compare the changes in behavior and cognitive function before and after the control and DDR conditions. Statistically significant condition $\times$ time interactions were found for repetitive behaviors $(F=18.7, d f=21$,

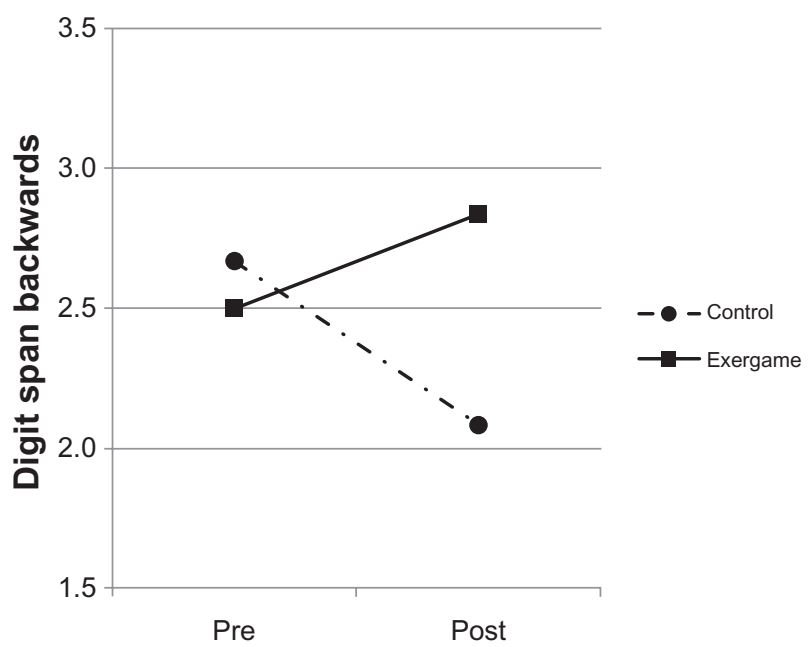

Figure I Change in executive function after exergaming compared with watching television.

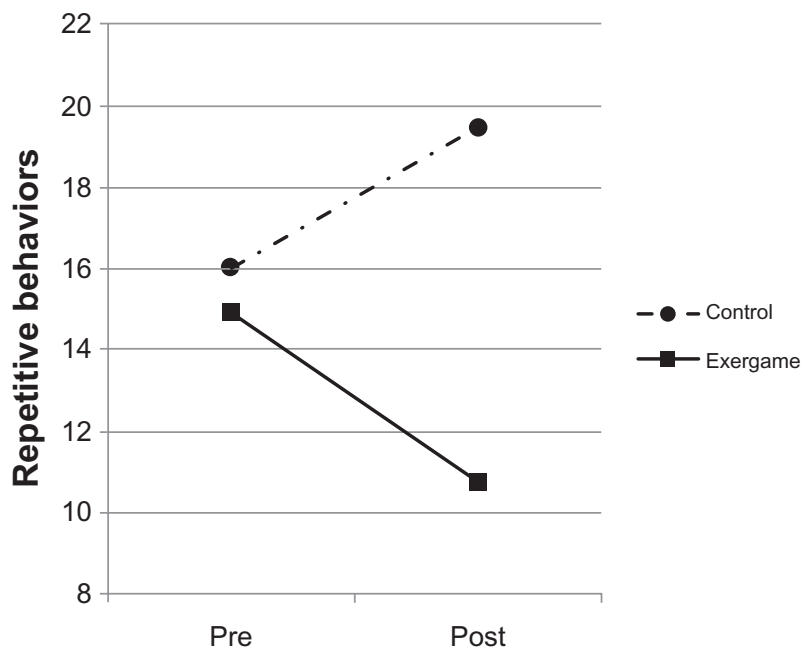

Figure 2 Change in repetitive behaviors after exergaming compared with watching TV.

$P<0.001, \eta \mathrm{p}^{2}=0.47$; Figure 1), Digits Backwards $(F=5.7$, $d f=22, P=0.03, \eta p^{2}=0.20$; Figure 2$)$, and Stroop C $(F=5.0$, $\left.d f=22, P=0.04, \eta p^{2}=0.19\right)$. Repetitive behaviors decreased significantly after exergaming compared with the control condition, while performance on Digits Backwards improved (means can be viewed in Table 1). Time to complete the Stroop $\mathrm{C}$ decreased for both the control and exercise condition, with the control condition improving the most which was likely due to the practice effects typically observed with the Stroop. ${ }^{40}$

\section{Pilot II: Cybercycling}

Data were analyzed from ten additional participants (mean age $=13.2$ years; $\mathrm{SD}=3.8$; range: $8-21$ years; all were male). The average score on the GARS-2 was $78.8(\mathrm{SD}=14.8)$.

Table I Neuropsychological and behavioral measures before and after exergaming and control condition

\begin{tabular}{|c|c|c|c|c|c|c|c|}
\hline & & \multicolumn{4}{|c|}{ Pilot I } & \multirow{2}{*}{\multicolumn{2}{|c|}{$\frac{\text { Pilot II }}{\text { Cybercycle }}$}} \\
\hline & & \multicolumn{2}{|c|}{ Control } & \multicolumn{2}{|l|}{ DDR } & & \\
\hline & & $M$ & $S D$ & $M$ & $S D$ & $M$ & $S D$ \\
\hline Digit span & Pre & 2.7 & $(1.61)$ & 2.5 & $(1.38)$ & 5.4 & $(2.17)$ \\
\hline Backwards & Post & 2.1 & (I.44) & 2.8 & (I.40) & 6.0 & $(2.91)$ \\
\hline \multirow[t]{2}{*}{ Stroop C } & Pre & 75.0 & $(23.4)$ & 54.9 & $(19.8)$ & 51.8 & $(17.8)$ \\
\hline & Post & 57.3 & $(I 7.6)$ & 51.0 & $(23.6)$ & 45.2 & $(12.9)$ \\
\hline \multirow[t]{2}{*}{ Color trails } & Pre & 182.5 & $(57.2)$ & I52.I & (50.9) & - & - \\
\hline & Post & 151.7 & $(40.8)$ & 146.6 & $(65.8)$ & - & - \\
\hline Repetitive & Pre & 16.0 & (9.79) & 14.9 & (8.58) & 9.7 & $(11.25)$ \\
\hline Behavior & Post & 19.5 & $(12.9)$ & 10.8 & (7.34) & 8.6 & $(9.27)$ \\
\hline
\end{tabular}

Note: Simple main effects were not hypothesized, but are reported for between group differences at post-test for exploratory purposes. Controls vs DDR at post: Digits Backwards $(P=0.21)$ and Repetitive Behaviors $(P=0.06)$. Controls versus cybercycle at post: Digits Backwards $(P<0.001)$ and Repetitive Behaviors $(P=0.05)$. The significant interactions reported in the text indicate that the relative change from pre to post in the intervention groups was significantly different from the change in the control group on these two variables.

Abbreviations: M, mean; SD, standard deviation; DDR, Dance Dance Revolution. 
There were no significant differences between the cybercycling group and the control condition (Pilot I) on age or baseline GARS-2. To assess the effect of the exercise intervention (cybercycle), three repeated measures analyses of variance were performed to compare changes in behavior and cognitive function in the control condition (Pilot I) and cybercycling (Pilot II). Statistically significant condition $\times$ time interactions were found for repetitive behaviors $(F=5.53, d f=20$, $\left.P=0.03, \eta p^{2}=0.24\right)$ and Digits Backwards $(F=5.59$, $\left.d f=20, P=0.03, \eta_{\mathrm{p}}{ }^{2}=0.22\right)$. Repetitive behaviors decreased significantly after cybercycling, while performance on Digits Backwards improved compared with the control condition (means can be viewed in Table 1).

\section{Discussion}

Twenty-two young persons with ASD were participants in two pilot studies in which change in behavioral and cognitive performances increased after exergaming (DDR or cybercycling) compared with change in performance after a control condition (watching a video). Significant interaction effects were found, revealing improvement after exergaming (for both DDR and cybercycling) compared with the control condition, for repetitive behaviors and one measure of executive function (Digits Backwards). These results, although preliminary, are encouraging since the repetitive behaviors characteristic of ASD provide an almost insurmountable barrier to social interaction and community integration. ${ }^{5}$ Furthermore, the positive impact of exergaming on executive function may lend additional cognitive control to students coping with repetitive behaviors of ASD. The results of these two pilot studies suggest exergaming may be a useful adjunct in the treatment of ASD and should prompt further investigation.

The findings reported herein are consistent with case studies and preliminary research that have shown that aerobic activity can decrease self-stimulatory behaviors in children with autism, ${ }^{15-17}$ and can also lead to improved performance on executive function tasks in normative youth. ${ }^{41}$ While executive function improved significantly only on Digits Backwards herein, similar gains were noted on the two remaining executive function tests (Color Trails 2 and Stroop C), but they did not reach significance compared with gains made in the control condition. This apparent discrepancy is most likely due to the increased burden on the exergaming condition of exceeding the large initial practice effects noted on these timed tests during the first control administration. ${ }^{40}$

One strength of the current study is that two different exergames were examined (DDR and cybercycling) and both were found to have similar positive effects on repetitive behaviors and executive function. It is noteworthy that both of the exergames utilized have greater aerobic activity potential (due to required quick large muscle movements) compared with other exergames like Wii (eg, bowling or tennis), which function primarily in response to hand and arm movements. Additionally, the study demonstrates feasibility of utilizing exergames with children with ASD. Since exergames are becoming more widely available and affordable, it may be possible to implement these interventions both at home and in schools.

\section{Limitations and directions for future research}

One important limitation of the current research is the small sample size, even though it is an improvement over the predominant prior use of case studies. With a small sample, a within-subjects design was more practical than randomly assigning participants to an intervention type. It would be beneficial for future research to include a randomized design with a larger sample. Additionally, it is unclear what components of the exergaming condition yielded the primary benefit (eg, aerobic exercise or mental challenge); it may be that exergaming served to motivate adequate exercise to produce an effect. A follow-up study could compare traditional exercise with exergaming and videogame play, which could tease apart the contributing factors behind behavioral and cognitive effects. Random ordering of the interventions in a withinsubjects design would strengthen the internal validity of the study. A recent study compared neurophysiological evidence of the cognitive effects on college students of a single bout of traditional exercise, exergaming, and videogame play, and found only traditional exercise yielded significant change. ${ }^{42}$ This points to the need for more well-designed comparative research to clarify the role of possible factors. Such additional information might guide the development of more refined interventions, perhaps allowing for greater precision in tailoring interventions to meet the needs of individual patients (eg, emphasizing behavioral change or cognitive control as most needed).

Another limitation of the current research is the lack of a quantitative measurement of exertion or exercise intensity which has in some cases been linked to the magnitude of effect. While participants in this study did complete the exergaming intervention for a full 20 minutes, it is possible that some participants exerted greater effort than others (eg, more frequent or vigorous dance steps), which could impact the magnitude of behavioral or cognitive outcomes. For example, Kern and colleagues ${ }^{43}$ found that in three children with ASD, 
15 minutes of vigorous exercise (jogging) was effective in decreasing behaviors while mild exercise (ball play) was not. Similarly, Levinson and Reid ${ }^{44}$ found that for three children, jogging was more effective than walking for reducing behaviors. Elliot and colleagues ${ }^{45}$ compared general motor training activities (eg, weight-training or walking) and vigorous aerobic exercise (jogging) as antecedent conditions for decreasing repetitive behaviors in adults with both autism and mental retardation. Vigorous, aerobic exercise was needed to significantly reduce repetitive behaviors. Celiberti and colleagues ${ }^{46}$ also compared walking and jogging as antecedents in a case study, and concluded that vigorous aerobic activity was more effective in producing the desired changes in behavior. Variability in some study outcomes may be due to variability in exercise intensity or duration across studies. For example, Oriel and colleagues ${ }^{47}$ used a within-subjects crossover design to examine the academic and behavioral effects of 15 minutes of jogging for four classrooms of children with ASD. They found academic responses improved, but not behaviors, so it may be that the amount of exercise was insufficient. Future research could quantify exertion in terms of intensity and duration, perhaps by measuring heart rate and energy expended (eg, with a pedometer or accelerometer).

It would also be interesting to explore the potential impact of longer-term regular exergaming over a period of weeks or months to see if it holds participants' interest as is predicted, given its entertaining and reinforcing nature. An examination of the duration of effects would be useful, since most research to date has only examined effects in the immediate period following exercise. Knowing if effects can be sustained or cumulate would add to the applicability of the findings. Furthermore, it is unclear if exergaming, DDR in particular, can impact cardiorespiratory fitness, ${ }^{48}$ thus fitness effects should be evaluated (eg, $\mathrm{VO}_{2}$ with a respirometer). Additionally, longer-term interventions could evaluate the role of mood, motivation, and other factors in adherence to exergaming as applied to ASD. Recent research suggests that parental and peer involvement appears to be key in sustaining use of DDR at home. ${ }^{49}$

Finally, it may be fruitful to investigate additional outcomes such as improved social skills. As noted above, $\operatorname{Pan}^{35}$ found improved social skills in 16 children with ASD after 10 weeks of group aquatic exercise. It would be interesting to examine the potential benefits of exergaming in the social realm for ASD, since it is possible to control a number of variables in an exergame which could moderate outcomes. For example, given the potential for modifying avatars representing the self and others in exergames with differential effects,${ }^{50}$ future research could explore a variety of social outcomes based on manipulations of avatars and also the social environment of an exergame (eg, individual versus collaborative versus competitive play).

\section{Implications}

Exergaming may be a useful adjunct to treatment for ASD, as it has been found in these pilot studies to decrease repetitive behaviors and increase executive function compared with a control condition. One application may be to encourage students with ASD to participate in a brief, 20-minute bout of exergaming, prior to engaging in educational or vocational tasks (and perhaps repeated at intervals throughout the day), since this may reduce repetitive behaviors and enhance executive control functions. Furthermore, the behavioral and cognitive results from the control group seem to indicate that sedentary leisure activity such as watching a television show actually increased repetitive behaviors and diminished executive function control. Thus, it may be advisable to limit the amount of time children with ASD are permitted to watch television programming. Interestingly, a recent study of DDR in the home found that it could simultaneously increase physical activity and decrease sedentary screen time. ${ }^{51}$

\section{Conclusion}

This research provides initial evidence that exergaming may be useful for the management of behavioral disturbance and for increasing cognitive control in children on the autism spectrum. Additional research should be conducted to replicate and extend these findings, to clarify exergaming components which may influence the behavioral and cognitive outcomes, and to examine the long-term and applied utility of exergaming for ASD.

\section{Acknowledgments}

This research was supported in part by student grants from the Internal Education Fund at Union College. We wish to thank the children, parents, and administrators of the Early Childhood Learning Center of New Jersey for their participation in this study. We also wish to thank the Autism Society of America - Albany Chapter, the Asperger's Syndrome Advocacy Group of Schenectady, Campbell House of Schenectady, Deborah Garrelts of ASPIRe NY, Inc, the National Autism Society - Albany Chapter, and the Parent to Parent program of New York State, for their assistance in recruiting participants for this study. The authors are grateful to the following research assistants for their work in data 
collection, video coding, and other aspects of the study: Lyndsay DeMatteo, Julia Dimitrova, Arielle Gartenberg, Ashley Gilmore, Ciro Griffiths, Stephanie Mugnai, Joseph Nimon, and Amanda Snyder.

\section{Disclosure}

The authors report no conflicts of interest in this work.

\section{References}

1. American Psychiatric Association. Diagnostic and Statistical Manual of Mental Disorders, 4th ed. - Text-Revised (DSM-IV-TR). Washington, DC: American Psychiatric Association; 2000.

2. Centers for Disease Control and Prevention. Prevalence of autism spectrum disorders - Autism and Developmental Disabilities Monitoring Network, 14 sites, United States, 2002. Surveillance Summaries. 2007;56(SS-01):12-28. Available at: http://www.cdc.gov/mmwr/ preview/mmwrhtml/ss5601a2.htm. Accessed August 24, 2011.

3. Happé F, Frith U. The neuropsychology of autism. Brain. 1996;119 (Pt 4):1377-1400.

4. Turner M. Repetitive behavior in autism: a review of psychological research. J Child Psychol Psychiatry. 1999;40(6):839-849.

5. Lord CE. Autism: from research to practice. Am Psychol. 2010;65(8):815-826.

6. Reichow B, Doehring P, Cicchetti D, Volkmar F, eds. Evidence-Based Practices and Treatments for Children with Autism. New York, NY: Springer; 2011.

7. Lopez BR, Lincoln AJ, Ozonoff S, Lai Z. Examining the relationship between executive functions and restricted, repetitive symptoms of autistic disorder. J Autism Dev Disord. 2004;35(4):445-460.

8. Lang R, Koegel L, Ashbaugh K, Regester A, Ence W, Smith W. Physical exercise and individuals with autism spectrum disorders: A systematic review. Res Aut Spectr Dis. 2010;4(4):565-576.

9. Petrus C, Adamson S, Block L, Einarson S, Sharifnejad M, Harris S. Effects of exercise interventions on stereotypic behaviours in children with autism spectrum disorder. Physiother Can. 2008;60(2):134-145.

10. Brisswalter J, Collardeau M, Rene A. Effects of acute physical exercise characteristics on cognitive performance. J Sports Med. 2002;32(9):555-566.

11. Colcombe $\mathrm{S}$, Kramer A. Fitness effects on the cognitive function of older adults: A meta-analytic study. Psychol Sci. 2003;14(2):125-130.

12. Hillman C, Belopolsky A, Snook E, Kramer F, McAuley E. Physical activity and executive control: Implications for increased cognitive health during older adulthood. Res Q Exerc Sport. 2004;75(2):176-185.

13. Colcombe SJ, Erickson KI, Scalf PE, et al. Aerobic exercise training increases brain volume in aging humans. J Gerontol A: Bio Sci Med Sci. 2006;61(11):1166-1170.

14. Erickson KI, Voss MW, Prakash RS, et al. Exercise training increases size of hippocampus and improves memory. Proc Natl Acad Sci U SA. 2011;108(7):3017-3022.

15. Watters RG, Watters WE. Decreasing self-stimulatory behavior with physical exercise in a group of autistic boys. J Autism Dev Disord. 1980;10(4):379-387.

16. Kern L, Koegel RL, Dyer K, Blew PA, Fenton LR. The effects of physical exercise on self-stimulation and appropriate responding in autistic children. J Autism Dev Disord. 1982;12(4):399-419.

17. Powers S, Thibadeau S, Rose K. Antecedent exercise and its effects on self-stimulation. Behavioral Residential Treatment. 1992;7(1):15-22.

18. Lochbaum MR, Crews DJ. Exercise prescription for autistic populations. J Autism Dev Disord. 1995;25(3):335-336.

19. Rosenthal-Malek A, Mitchell S. Brief report: the effects of exercise on the self-stimulatory behaviors and positive responding of adolescents with autism. J Autism Dev Disord. 1997;27(2):193-202.
20. Pan C. Effects of water exercise swimming program on aquatic skills and social behaviors in children with autism spectrum disorders. Autism. 2010;14(1):9-28.

21. Fragala-Pinkham M, Haley S, O'Neil M. Group swimming and aquatic exercise programme for children with autism spectrum disorders: a pilot study. Dev Neurorehabil. 2011;14(4):230-241.

22. Owen AM, Hampshire A, Grahn JA, et al. Putting brain training to the test. Nature. 2010;465(7299):775-778.

23. Grynszpan O, Perbal S, Pelissolo A, et al. Efficacy and specificity of computer-assisted cognitive remediation in schizophrenia: A metaanalytical study. Psychol Med. 2011;41(1):163-173.

24. Wykes T, Huddy V, Cellard C, McGurk S, Czobor P. A meta-analysis of cognitive remediation for schizophrenia: methodology and effect sizes. Am J Psychiatry. 2011;168(5):472-485.

25. Durkin K. Videogames and young people with developmental disorders. Rev Gen Psychol. 2010;14(2):122-140.

26. Standen PJ, Brown DJ. Virtual reality in the rehabilitation of people with intellectual disabilities: Review. CyberPsychol Behav [serial on the Internet]. 2005;8(3):272-282.

27. Trepagnier CY, Sebrechts MM, Finkelmeyer AA, Coleman MM, Stewart WR, Werner-Adler MM. Virtual environments to address autistic social deficits. Ann Rev CyberTher Telemed [serial on the Internet]. 2005;3:101-107.

28. Lieberman DA. Designing serious games for learning and health in informal and formal settings. In: Ritterfeld U, Cody M, Vorderer V, editors. Serious Games: Mechanisms and Effects. New York, NY: Routledge; 2009:117-130.

29. Hayes E, Silberman L. Incorporating video games into physical education. $J$ Phys Educ Recr Dance [serial on the Internet]. 2007;78(3):18-24.

30. Graf D, Pratt L, Hester C, Short K. Playing active video games increases energy expenditure in children. Pediatrics [serial on the Internet]. 2009;124(2);534-540.

31. Lotan M, Yalon-Chamovitz S, Weiss P. Improving physical fitness of individuals with intellectual and developmental disability through a virtual reality intervention program. Res Dev Disabil. 2009;30(2):229-239.

32. Mhurchu CN, Maddison R, Jiang Y, Jull A, Prapavessis H, Rodgers A. Couch potatoes to jumping beans: A pilot study of the effects of active video games on physical activity in children. Int J Behav Nutr Phys Act. 2008;5:8.

33. Yim J, Graham TC. Using games to increase exercise motivation. Future Play. 2007:166-173.

34. Leininger L, Coles M, Gilbert J. Comparing enjoyment and perceived exertion between equivalent bouts of physically interactive video gaming and treadmill walking. Health Fitness J Canada. 2010;3(1): 12-18.

35. Pan C, Frey G. Physical activity patterns in youth with autism spectrum disorders. J Autism Dev Disord. 2006;36(5):597-606.

36. Gilliam J. GARS-2: Gilliam Autism Rating Scale. 2nd ed. Austin, TX: PRO-ED; 2006.

37. Lezak MD, Howieson DB, Loring DW, Hannay H, Fischer JS. Neuropsychological Assessment. 4th ed. New York, NY: Oxford University Press; 2004.

38. D'Elia LF, Satz P, Uchiyama CL, White T. Color Trails Test. Odessa, FL: PAR, Inc; 1996.

39. van der Elst W, van Boxtel MJ, van Breukelen GP, Jolles J. The Stroop color-word test: influence of age, sex, and education; and normative data for a large sample across the adult age range. Assessment. 2006;13(1):62-79.

40. Beglinger L, Gaydos B, Tangphao-Daniels O, et al. Practice effects and the use of alternate forms in serial neuropsychological testing. Arch Clin Neuropsychol. 2005;20(4):517-529.

41. Tomporowski PD. Cognitive and behavioral responses to acute exercise in youths: a review. Pediatr Exerc Sci. 2003;15(4):348-359. 
42. O’Leary K, Pontifex M, Scudder M, Brown M, Hillman C. The effects of single bouts of aerobic exercise, exergaming, and videogame play on cognitive control. Clin Neurophysiol. 2011;122(8):1518-1525.

43. Kern L, Koegel RL, Dunlap G. The influence of vigorous versus mild exercise on autistic stereotyped behaviors. J Autism Dev Disord. 1984;14(1):57-67.

44. Levinson LJ, Reid G. The effects of exercise intensity on the stereotypic behaviors of individuals with autism. Adapt Phys Activ $Q$. 1993;10(3):255-268.

45. Elliott RO, Dobbin AR, Rose GD, Soper HV. Vigorous, aerobic exercise versus general motor training activities: Effects on maladaptive and stereotypic behaviors of adults with both autism and mental retardation. J Autism Dev Disord. 1994;24(5):565-576.

46. Celiberti DA, Bobo HE, Kelly KS, Harris SL, Handleman JS. The differential and temporal effects of antecedent exercise on the self-stimulatory behavior of a child with autism. Res Dev Disabil. 1997;18(2):139-150.
47. Oriel K, George C, Peckus R, Semon A. The effects of aerobic exercise on academic engagement in young children with autism spectrum disorder. Pediatr Phys Ther. 2011;23(2):187-193.

48. Unnithan V, Houser W, Fernhall B. Evaluation of the energy cost of playing a dance simulation video game in overweight and non-overweight children and adolescents. Int J Sports Med. 2006;27(10):804-809.

49. Paez S, Maloney A, Kelsey K, Wiesen C, Rosenberg A. Parental and environmental factors associated with physical activity among children participating in an active video game. Pediatr Phys Ther. 2009;21(3):245-253.

50. Jin S. Avatars mirroring the actual self versus projecting the ideal self: The effects of self-priming on interactivity and immersion in an exergame, Wii Fit. CyberPsychol Behav [serial on the Internet]. 2009;12(6):761-765.

51. Maloney AE, Bethea T, Kelsey KS, et al. A pilot of a video game (DDR) to promote physical activity and decrease sedentary screen time. Obesity (Silver Spring). 2008;16(9):2074-2080.

\section{Publish your work in this journal}

Psychology Research and Behavior Management is an international, peerreviewed, open access journal focusing on the science of psychology and its application in behavior management to develop improved outcomes in the clinical, educational, sports and business arenas. Specific topics covered include: Neuroscience, memory \& decision making; Behavior

\section{Dovepress}

modification \& management; Clinical applications; Business \& sports performance management; Social and developmental studies; Animal studies. The manuscript management system is completely online and includes a quick and fair peer-review system. Visit http://www.dovepress. com/testimonials.php to read real quotes from published authors.

Submit your manuscript here: http://www.dovepress.com/psychology-research-and-behavior-management-journal 